\title{
Tools for Dark Matter in Particle and Astroparticle Physics
}

\section{Alexander Pukhov*}

Skobeltsyn Inst. of Nuclear Physics, Moscow State Univ., Moscow, Russia

E-mail: pukhov@lapp.in2p3.fr

\section{Geneviève Bélanger}

LAPTH, Univ. de Savoie, CNRS, B.P.110, F-74941 Annecy-le-Vieux,France

E-mail: belanger@lapp.in2p3.fr

\section{Fawsi Boudjema}

LAPTH, Univ. de Savoie, CNRS, B.P.110, F-74941 Annecy-le-Vieux, France

E-mail: boudjema@lapp.in2p3.fr

\section{Andrei Semenov}

Joint Institute for Nuclear Research (JINR) 141980, Dubna, Russia

E-mail: semenov@lapp.in2p3.fr

Despite several indirect confirmations of the existence of dark matter, the properties of a new dark matter particle are still largely unknown. Several experiments are currently searching for this particle underground in direct detection, in space and on earth in indirect detection and the LHC. A confirmed signal could select a model for dark matter among the many extensions of the standard model. In this paper we present a short review of the public codes for computation of dark matter observables.

13th International Workshop on Advanced Computing and Analysis Techniques in Physics Research February 22-27, 2010

Jaipur, India

\footnotetext{
${ }^{*}$ Speaker.
} 


\section{Introduction}

Nowadays there are two crucial problems in particle physics: the search for the Higgs particle or more generally the unravelling of the mechanism of symmetry breaking and the nature of Dark Matter (DM). The Higgs particle, responsible for symmetry breaking, is the cornerstone of the Standard Model (SM) and of some of its extensions. As long as the Higgs particle escapes detection there will be a missing link in our understanding of the nature of fundamental interactions and the SM will be incomplete. As concerns the nature of DM we are facing a different issue: there is robust experimental evidence for DM, yet we have no additional evidence for the existence of a stable massive particle which can play the role of DM. For this we have to consider extensions of the SM and assume an additional discrete unbroken symmetry, for example $Z_{2}$. This symmetry not only allows the lightest particle of the new physics model to be stable but it also usually makes this model conform more naturally with current data.

Let's review briefly the experimental evidence for dark matter. First, the radial dependence of rotation curves of galaxies give strong evidence for DM. Typical rotation curves in spiral galaxies show a plateau at a distance of several kpc from the galactic center, see Fig1(a). The numerical value of the velocity at large distances is significantly larger than expected assuming only visible matter. Furthermore such a plateau implies a gravitational mass that increases linearly with the galactic radius, this does not corresponds to the distribution of visible matter. The rotation curve for the Milky Way allows to estimate the density of DM in the Sun orbit. This gives a value of $\rho_{D M} \approx 0.3 \mathrm{GeV} / \mathrm{cm}^{3}$ for the local density. This number enters the computation of the signals for DM direct and indirect detection that will be described below.
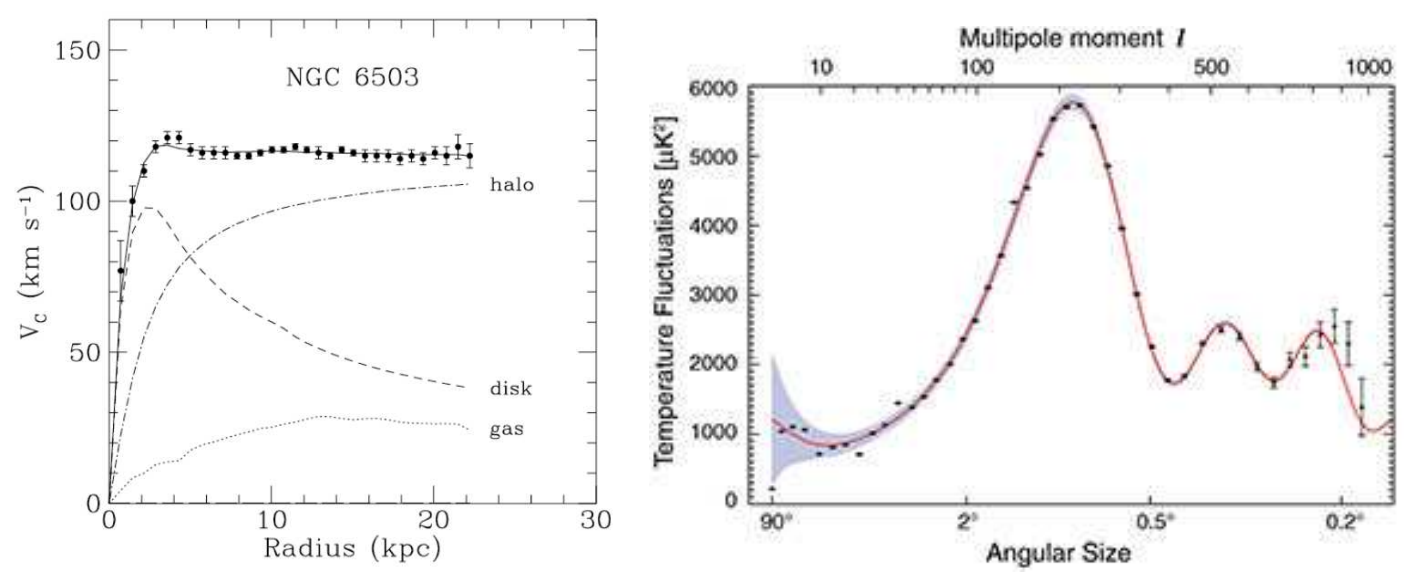

Figure 1: a) Measured rotation curve for the N6503 galaxy together with estimations of the contribution of the halo, disk and galactic gas to the rotation velocity (dashed). b) Temperature fluctuations measured by WMAP. The DM density is extracted from the second peak.

Very precise estimates of the amount of DM were obtained from the WMAP measurement of fluctuations in the microwave background temperature[1, 2]. Temperature fluctuations, Fig1(b), are connected to fluctuations in the gravitational potential in the time of last scattering. Because ordinary matter is in a plasma at high temperatures, it can not generate such fluctuations. Precise numerical analyses of WMAP results allow to extract the total density of DM particles in the time 
of last scattering. Assuming that the number of DM particles has not changed since then we should have now a DM density around $1 \mathrm{GeV} / \mathrm{m}^{3}$. More precisely,

$$
\Omega h^{2}=\frac{\rho_{D M}}{\rho_{c}}=0.1099 \pm 0.0062
$$

where $\rho_{c}$ is the critical density. This amount of DM is in good agreement with simulations of large structure formation in Universe. Indeed baryonic matter itself is not able to create galaxies because of the fast expansion rate of the Universe. Because DM particles have to be non-relativistic at the time of last scattering time, from WMAP measurements one can estimate its mass to be $M_{D M}>10 \mathrm{KeV}$. This is sufficient to rule out neutrinos as the main component of DM. Therefore it is necessary to extend the SM to explain the nature of DM. The precise measurement of $\Omega h^{2}$ provides a powerful mean to discriminate various extensions of the SM that propose a DM candidate.

There are three kinds of astroparticle experiments which allow in principle to detect DM particles and measure some of their properties. First, experiments for indirect DM detection such as PAMELA [3, 6], HEAT [4], AMS01 [5], Fermi [7, 15], ATIC [8], HESS [9, 13, 14] INTEGRAL [10], Veritas[11], EGRET[12] try to observe the products of DM self annihilation in the galactic halo. The SM particles that are produced in this annihilation will decay to stable particles including $\gamma, e^{ \pm}, p(\bar{p})$ and neutrinos. Indirect detection experiments search primarily for $e^{+}, \bar{p}, \gamma$ as the $e^{-}$ and $p$ channels suffer from a very large background and the neutrino signal is expected to be low. Interpreting the results of indirect detection experiments requires a good understanding of both the background caused by galactic sources as well as the structure of galactic magnetic fields responsible for the propagation of $e^{+}$and $\bar{p}$. For instance, the excess of positrons recently observed by PAMELA can be caused either by some exotic DM or some galactic source like supernova. Furthermore large uncertainties in the signal can be caused by a clumpy structure in the DM distribution (this can increase the signal by a factor 20).

Direct detection experiments such as Edelweiss[16], DAMA[17], CDMS[18, 20], Xenon [19, 21], Zeplin[22] or Cogent[23] measure the recoil energy of the nuclei that would result from an elastic DM - nucleus collision in a large detector. To reduce the cosmic rays background such detectors are located deep underground. We should mention that DAMA has for several years found a positive result, such a signal has not yet been confirmed by other experiments [17].

High energy neutrinos produced as a result of the annihilation of DM particles captured in the center of the Sun and the Earth, are searched by Super-Kamiokande [24], Antares [25] or IceCube [26]. The rate of DM annihilation inside the Sun/Earth should be equal to the rate of DM capture by the Sun/Earth. These experiments are therefore similar to direct detection experiments where the Sun or the Earth plays the role of the large detector. These experiments have not yet observed DM events.

Dark matter can also be detected in accelerators such as the Tevatron or the LHC. Despite the fact that the direct production of DM particles has a small cross section and that the DM particle escapes the detector without leaving a track, a DM particle could be detected at LHC. Indeed such particle appears in the decay chains of new particles that can be directly produced at a collider and its signature is a large amount of missing energy [30]. It is therfore possible that the LHC will soon shed light on the two fundamental problems in particle physics: Higgs and dark matter. 


\section{Short review of theoretical models for DM}

Many extensions of the SM that can provide a DM candidate have been proposed. The best studied and most popular models among these are supersymmertic models: the minimal supersymmetric model MSSM [32, 31] and its extensions such as the NMSSM [33] and the CPVMSSM [34]. In these models R-parity conservation guarantees the stability of the DM particle. In models with flat or warped extra dimensions [37, 38], some parity that depend on the field in extra dimensions is responsible for the stability of DM. Furthermore there are models with extended gauge or Higgs sectors [42, 43] as well as little Higgs models [39, 40, 41]. In these models the DM candidate can be either a Majorana fermion, a Dirac fermion, a vector boson or a scalar.

\section{Calculations needed for DM analyses.}

Because of the large number of astroparticle experiments and the large number of theoretical models we need software tools for the computation of DM properties and DM detection rates in different experiments. The general theoretical formulas for DM calculations are available in the review [27]. Detailed relic density calculations in the MSSM can be found in [28, 29] while direct detection formulas including loop corrections and subleading terms was obtained in [36]. The different tasks which have to be solved are

- Calculation of DM relic density. The formalism to calculate the DM density using the freezeout mechanism and based on the DM annihilation cross sections was developed in [28, 29]. One has to solve a differential equation that gives the temperature dependence of DM density. The dependence on the underlying model appears via the calculation of the thermally averaged cross section for DM annihilation. A rough estimation gives a value for the annihilation cross section around $\sigma(\mathrm{v}) \mathrm{v} \approx 1 \mathrm{pb} \cdot \mathrm{c}$. This corresponds to a typical weak interaction cross section. Nevertheless agreement with WMAP results strongly constrain the parameters of the particle physics model. In addition to DM anihilation processes, processes involving other particles that are odd under the discrete symmetry and whose masses are just above that of DM also contribute to the effective cross section since eventually all these particles will decay into the DM and some other particles. The large number of processes involved and the fact that a priori the matrix elements needed are not known means that relic density calculations in a generic model of Dm can be challenging.

- Calculation of DM - nucleon cross sections. These are required for the prediction of rates in direct detection and neutrino telescope experiments. In the standard case one needs to compute the DM-nuclei scattering amplitude in the limit of small momentum transfer. This is obtained from the DM-nucleon amplitude which is in turn related to DM-quark amplitudes.

- Calculation of indirect detection signal. In addition to the calculation of the DM annihilation cross section, the computation of the spectra of photons, positrons and antiprotons are required. The initial spectra can be easily obtained even for a generic model. For this one has to calculate all $2 \rightarrow 2$ annihilation cross sections and extract the $\gamma, e^{+}$and $\bar{p}$ spectra using Pythia. The $2 \rightarrow 2+\gamma$ processes also might have to be taken into account[35]. The 
propagation of $e^{+}$and $\bar{p}$ can be done by solving the diffusion equation by the Green function method. A more precise treatment as well as the computation of the background require a Monte Carlo simulation.

- Calculation of low energy constraints. Several experimental data restrict the parameter space of SM extensions even though they are not directly related to DM observables. These include precision measurements such as the muon anomalous magnetic moment, $g-2$, or rare $B$ decays for example $b \rightarrow s \gamma, B_{s} \rightarrow \mu^{+} \mu^{-}$. In most cases the theoretical prediction require the computation of higher order processes involving Feynman diagrams at the loop level. These are not completely automatized yet.

- LEP and Tevatron constraints. High energy collider experiments are probing the SM and its extensions. Their results can be used to put constraints on the Higgs mass, on new channels in $\mathrm{Z}$ decay and on the mass of heavy exotic particles including the supersymmetric partners of SM particles.

- Calculation of LHC and ILC signals The computation of signals associated with new particles produced at colliders include the matrix element calculation for the production and for the decays of the new particles as well as Monte Carlo phase space integration and cuts implementation. Several tools have ben developed to perform these tasks.

\section{Review of software for DM calculation}

There are several public codes used in DM calculations which were designed for the study of physics beyond the SM, for a review of the different tools see [53, 54]. Several codes perform the computation of the particle mass spectrum in supersymmetric models, indeed large loop corrections are generic and need to be taken into account. These codes also solve the renormalization group equations in supersymmetric scenarios with fundamental parameters defined at the GUT scale. Four codes were developed in the framework of the MSSM, SoftSUSY [47], Isajet [58, 57] and SPheno [46], while NMSSMTools [49] and CPsuperH[45] deal with extensions of the MSSM. These codes also compute various low energy and collider constraints. A special file interface SLHA [51, 52] was designed for these programs. This interface facilitates their use in DM related code. The package HiggsBounds [50] was designed for testing LEP and Tevatron accelerator constraints on the Higgs sector in generic models. Such constraints are available in NMSSMTools and Isajet but only for the specific class of models they support.

A very important tool for analysis of indirect detection experiments is the GALPROP[55] program. It gives a numerical solution for the differential equation that describes the propagation of different kind of particles in the galactic magnetic fields. Although this code is rather slow it allows to take into account both DM signals and background galactic sources at the same time.

There are four public codes for DM studies in supersymmetry, SuperIso[59], IsaTools [58, 57] DarkSUSY [56] and micrOMEGAs [60, 61, 62]. All perform the computation of the DM relic density together with other observables that are not necessarily related to DM. SuperIso is a rather new code that is primarily dedicated to flavour physics in the MSSM, the SLHA is used for interfacing spectrum calculators. IsaTools and DarkSUSY were also both developed for the 
MSSM. They calculate the direct detection and indirect detection rates as well as low energy and accelerator constraints. IsaTools uses Isajet to compute the particle spectrum while DarkSUSY also uses SuSpect or the SLHA. DarkSUSY also calculates the neutrino rates from DM annihilation in the Sun and the Earth, furthermore DarkSUSY includes the propagation of cosmic rays. In particular it is interfaced with GALPROP which allows to study both signal and background in indirect detection measurements. For MSSM applications, DarkSUSY is now the most complete package. On the other hand IsaTools is based on Isajet, a tool for computation of signals for SM and its supersymmetric extensions at colliders and is therfore most suited for DM accelerator studies.

micrOMEGAs is the only package for DM studies in generic extensions of the SM model. Details of the techniques used in micrOMEGAs are explained in the next section. micrOMEGAs computes the DM relic density, direct detection and indirect detection rates. For the propagation of $e^{+}$and $\bar{p}$, micrOMEGAs uses a Green function method which describes well the signals from DM annihilation but does not allow to calculate the background. The neutrino rate from capture in celestial bodies is not yet implemented in micrOMEGAs. Low energy and collider constraints are provided for some models and the predictions of collider signals are obtained from CalCHEP which is included in micrOMEGAs. The current version of micrOMEGAs contains the MSSM, NMSSM, CPVMSSM, the Little Higgs model [41], and a Dirac Neutrino DM model [44].

Comparisons of IsaTools/DarkSUSY/micrOMEGAs showed good agreement between the codes. In fact such cross checks were used to remove several bugs in these packages.

\section{Applications of automatic matrix element calculators for dark matter studies.}

In principle it is not necessary to use only one universal program to study DM properties in any model. On the other hand once such a tool has been tested and debugged for one specific extension of the SM, it can rapidly and straightforwardly be used for other models as well. An automatic approach therfore increases the reliability of the software and considerably reduces the time needed for developing new software as well as the time required for the user to become familiar with a new package.

As mentionned above the most important computer task needed for DM studies is the computation of matrix elements of various reactions which occur in some specific model of particle physics. In the last years several automatic calculators of matrix elements were developed: CompHEP [63], CalcHEP[64], FeynArts/FormCalc [65, 66, 67], MadGraph[68, 69], Sherpa[70], and Omega [71]. In principle any of these could be used for DM related calculations in a generic model. Currently the idea of automatic matrix element generation for DM observables in a generic model is realized in full scope only in the micrOMEGAs package. This approach was first applied for the computation of the relic density [60]. In [61] a numerical algorithm for the calculation of the spin-dependent and spin-independent DM-nucleon amplitudes relevant for direct detection was proposed and implemented. This algorithm which can be applied to a generic model replaces the usual symbolic computation of amplitudes by means of Fiertz identities. Recently in [62] an automatic approach for calculating the spectra of DM self annihilation in the galaxy was designed and takes into account processes with additional photon radiation [35].

The key point in micrOMEGAs' approach to DM calculations is the generation of shared libraries with matrix element codes. The calculation of all matrix elements that enter a relic density 
calculation is computer time consuming and requires a lot of disk space [56]. However for any particular set of model parameters in general only a small number of annihilation channels are needed. micrOMEGAs therefore generates the code only for the channels as they are needed, links them dynamically and stores them on the disk for subsequent usage.

Note that the idea of using automatic calculators in DM codes was also realised in IsaTools and SuperIso albeit only in the context of the MSSM. In IsaTools, CompHEP was used for generating (co-)annihilation cross sections while SuperIso relies on FeynCalc to evaluate the cross sections. In principle both these codes can be generalized for other models.

\section{Conclusion}

Several tools for the calculation of DM properties and DM signals for current and future experiments are now available. The currently most developed codes are DarkSUSY and micrOMEGAs. The existence of several independent codes is very important for cross checking the results and for understanding uncertainties which result from different technical implementation of the same algorithms. There are several auxiliary tools designed for the computation of the particle spectra and couplings as well as for calculation of low energy and high energy constraints. The development of interface protocols for data exchange between such programs is needed.

\section{Acknowledgments}

This work was supported in part by the GDRI-ACPP of CNRS, by the ANR project ToolsDMColl, BLAN07-2-194882, by the Russian foundation for Basic Research, RFBR-08-02-92499-a, RPBR-10-02-01443-a and by a State contract No.02.740.11.0244. The visit of A.P. to Jaipur was funded by the organizing committee and by the grant RFBR-10-07-08004-z.

\section{References}

[1] C. L. Bennett et al. [WMAP Collaboration], First Year Wilkinson Microwave Anisotropy Probe (WMAP) Observations: Preliminary Maps and Basic Results, Astrophys. J. Suppl. 148, 1 (2003).

[2] D. N. Spergel et al. [WMAP Collaboration], First Year Wilkinson Microwave Anisotropy Probe (WMAP) Observations: Determination of Cosmological Parameters, Astrophys. J. Suppl. 148, 175 (2003) [arXiv:astro-ph/0302209].

[3] O. Adriani et al. [PAMELA Collaboration], An anomalous positron abundance in cosmic rays with energies 1.5-100 GeV, Nature 458, 607 (2009) [arXiv:0810.4995 [astro-ph]].

[4] J. J. Beatty et al., New measurement of the cosmic-ray positron fraction from $5 \mathrm{GeV}$ to $15 \mathrm{GeV}$, Phys. Rev. Lett. 93, 241102 (2004) [arXiv:astro-ph/0412230].

[5] M. Aguilar et al. [AMS-01 Collaboration], Cosmic-ray positron fraction measurement from $1 \mathrm{GeV}$ to $30 \mathrm{GeV}$ with AMS-01, Phys. Lett. B 646, 145 (2007) [arXiv:astro-ph/0703154].

[6] O. Adriani et al., A new measurement of the antiproton-to-proton flux ratio up to $100 \mathrm{GeV}$ in the cosmic radiation, Phys. Rev. Lett. 102, 051101 (2009) [arXiv:0810.4994 [astro-ph]].

[7] A. A. Abdo et al. [Fermi LAT Collaboration], Measurement of the Cosmic Ray e+ plus e- spectrum from $20 \mathrm{GeV}$ to $1 \mathrm{TeV}$ with the Fermi Large Area Telescope, Phys. Rev. Lett. 102, 181101 (2009). 
[8] J. Chang et al., An Excess Of Cosmic Ray Electrons At Energies Of 300-800 Gev, Nature 456, 362 (2008).

[9] F. Aharonian et al. [H.E.S.S. Collaboration], Probing the ATIC peak in the cosmic-ray electron spectrum with H.E.S.S, Astron. Astrophys. 508, 561 (2009) [arXiv:0905.0105 [astro-ph.HE]].

[10] A. W. Strong et al., Gamma-ray continuum emission from the inner Galactic region as observed with INTEGRAL/SPI, Astron. Astrophys. 444, 495 (2005) [arXiv:astro-ph/0509290].

[11] G. Maier [VERITAS Collaboration], Observation of Galactic Gamma-ray Sources with VERITAS, AIP Conf. Proc. 1085, 187 (2009) [arXiv:0810.0515 [astro-ph]].

[12] D. J. Thompson, Gamma ray astrophysics: the EGRET results, Rept. Prog. Phys. 71, 116901 (2008).

[13] F. Aharonian et al. [H.E.S.S. Collaboration], Discovery of Very-High-Energy Gamma-Rays from the Galactic Centre Ridge, Nature 439, 695 (2006) [arXiv:astro-ph/0603021].

[14] F. Aharonian et al. [HESS collaboration], H.E.S.S. upper limit on the very high energy gamma-ray emission from the globular cluster 47 Tucanae, arXiv:0904.0361 [astro-ph.HE].

[15] C. Meurer [Fermi LAT Collaboration], Dark Matter Searches with the Fermi Large Area Telescope, AIP Conf. Proc. 719, 1085 (2009) [arXiv:0904.2348 [astro-ph.HE]].

[16] R. Lemrani [EDELWEISS Collaboration], Search for dark matter with EDELWEISS: Status and future, Phys. Atom. Nucl. 69, 1967 (2006).

[17] R. Bernabei et al., DAMA investigations on dark matter at Gran Sasso: Results and perspectives, AIP Conf. Proc. 878, 91 (2006).

[18] D. S. Akerib et al. [CDMS Collaboration], CDMS, supersymmetry and extra dimensions, Nucl. Phys. Proc. Suppl. 173, 95 (2007) [arXiv:astro-ph/0609189].

[19] J. Angle et al. [XENON Collaboration], First Results from the XENON10 Dark Matter Experiment at the Gran Sasso National Laboratory, Phys. Rev. Lett. 100, 021303 (2008).

[20] Z. Ahmed et al. [The CDMS-II Collaboration], Results from the Final Exposure of the CDMS II Experiment, arXiv:0912.3592 [astro-ph.CO].

[21] E. Aprile et al. [XENON100 Collaboration], First Dark Matter Results from the XENON100 Experiment, arXiv:1005.0380 [astro-ph.CO].

[22] T. Sumner [UKDMC Collaboration], Direct Dark Matter Searches: Drift And Zeplin, PoS HEP2005, 003 (2006).

[23] C. E. Aalseth et al., Results from a Search for Light-Mass Dark Matter with a P-type Point Contact Germanium Detector, arXiv:1002.4703 [astro-ph.CO].

[24] S. Desai et al. [Super-Kamiokande Collaboration], Search for dark matter WIMPs using upward through-going muons in Super-Kamiokande, Phys. Rev. D 70, 083523 (2004) [Erratum-ibid. D 70, 109901 (2004)] [arXiv:hep-ex/0404025].

[25] J.P. Ernenwein [ANTARES Collaboration], Indirect dark matter search with the ANTARES neutrino telescope, PoS IDM2008 (2008) 036.

[26] C. DeClercq et al. [IceCube Collaboration], Search for dark matter with the AMANDA and IceCube neutrino detectors, PoS IDM2008 (2008) 034.

[27] G. Jungman, M. Kamionkowski and K. Griest, Supersymmetric dark matter, Phys. Rept. 267 (1996) 195 [arXiv:hep-ph/9506380]. 
[28] G. B. Gelmini, P. Gondolo and E. Roulet, Neutralino dark matter searches, Nucl. Phys. B 351, 623 (1991).

[29] J. Edsjo and P. Gondolo, Neutralino Relic Density including Coannihilations, Phys. Rev. D 56, 1879 (1997) [arXiv:hep-ph/9704361].

[30] H. Baer, X. Tata, Dark matter and the LHC, arXiv:0805.1905 [hep-ph].

[31] H. Goldberg, Constraint on the photino mass from cosmology, Phys. Rev. Lett. 50, 1419 (1983) [Erratum-ibid. 103, 099905 (2009)].

[32] J. R. Ellis, J. S. Hagelin, D. V. Nanopoulos, K. A. Olive and M. Srednicki, Supersymmetric relics from the big bang, Nucl. Phys. B 238, 453 (1984).

[33] U. Ellwanger, C. Hugonie and A. M. Teixeira, The Next-to-Minimal Supersymmetric Standard Model, arXiv:0910.1785 [hep-ph].

[34] A. Pilaftsis, CP-odd tadpole renormalization of Higgs scalar-pseudoscalar mixing, Phys. Rev. D 58, 096010 (1998) [arXiv:hep-ph/9803297].

[35] T. Bringmann, L. Bergstrom and J. Edsjo, New Gamma-Ray Contributions to Supersymmetric Dark Matter Annihilation, JHEP 0801, 049 (2008) [arXiv:0710.3169 [hep-ph]].

[36] M. Drees and M. Nojiri, Neutralino-Nucleon Scattering Revisited, Phys. Rev. D 48, 3483 (1993).

[37] H. C. Cheng, K. T. Matchev and M. Schmaltz, Radiative corrections to Kaluza-Klein masses, Phys. Rev. D 66, 036005 (2002) [arXiv:hep-ph/0204342].

[38] K. Agashe and G. Servant, Warped unification, proton stability and dark matter, Phys. Rev. Lett. 93, 231805 (2004) [arXiv:hep-ph/0403143].

[39] J. Hubisz and P. Meade, Phenomenology of the little Higgs with T-parity, Phys. Rev. D 71, 035016 (2005) [arXiv:hep-ph/0411264].

[40] A. Martin, Dark matter in the simplest little Higgs model, arXiv:hep-ph/0602206.

[41] A. Belyaev, C. R. Chen, K. Tobe and C. P. Yuan, Phenomenology of littlest Higgs model with T-parity: Including effects of T-odd fermions, Phys. Rev. D 74 (2006) 115020 [arXiv:hep-ph/0609179].

[42] J. McDonald, Gauge Singlet Scalars as Cold Dark Matter, Phys. Rev. D 50, 3637 (1994).

[43] V. Barger et al., Recoil detection of the lightest neutralino in MSSM singlet extensions, Phys. Rev. D 75, 115002 (2007) [arXiv:hep-ph/0702036].

[44] G. Belanger, A. Pukhov and G. Servant, Dirac Neutrino Dark Matter, JCAP 0801009 (2008).

[45] J. S. Lee et al., CPsuperH: A computational tool for Higgs phenomenology in the minimal supersymmetric standard model with explicit CP violation, Comput. Phys. Commun. 156 (2004) 283.

[46] W. Porod, SPheno, a program for calculating supersymmetric spectra, SUSY particle decays and SUSY particle production at e+e-colliders, Comput. Phys. Commun. 153 (2003) 275.

[47] B. C. Allanach, SOFTSUSY: A C++ program for calculating supersymmetric spectra, Comput. Phys. Commun. 143, 305 (2002).

[48] A. Djouadi, J. L. Kneur and G. Moultaka, SuSpect: A Fortran code for the supersymmetric and Higgs particle spectrum in the MSSM, Comput. Phys. Commun. 176, 426 (2007) [arXiv:hep-ph/0211331].

[49] U. Ellwanger and C. Hugonie, NMSPEC: A Fortran code for the sparticle and Higgs masses in the NMSSM with GUT scale boundary conditions, Comput. Phys. Commun. 177 (2007) 399. 
[50] P. Bechtle et al., HiggsBounds: Confronting Arbitrary Higgs Sectors with Exclusion Bounds from LEP and the Tevatron, Comput. Phys. Commun. 181 (2010) 138.

[51] P. Skands et al., SUSY Les Houches Accord: Interfacing SUSY Spectrum Calculators, Decay Packages, and Event Generators, JHEP 0407, 036 (2004) [arXiv:hep-ph/0311123].

[52] B. Allanach et al., SUSY Les Houches Accord 2, Comput. Phys. Commun. 180 (2009) 8.

[53] P. Z. Skands et al., A repository for beyond-the-standard-model tools, http://www.ippp.dur.ac.uk/BSM/

[54] F. Boudjema, J. Edsjo, P. Gondolo, in Particle Dark Matter: Observations, Models and Searches, ed. G. Bertone, Cambridge University Press (2010) arXiv:1003.4748.

[55] A. W. Strong et al., The GALPROP Cosmic-Ray Propagation Code, arXiv:0907.0559 [astro-ph.HE].

[56] P. Gondolo et al., DarkSUSY: Computing supersymmetric dark matter properties numerically, JCAP 0407 (2004) 008 [arXiv:astro-ph/0406204].

[57] H. Baer, C. Balazs and A. Belyaev, Neutralino relic density in minimal supergravity with co-annihilations, JHEP 0203 (2002) 042 [arXiv:hep-ph/0202076].

[58] H. Baer, A. Belyaev, T. Krupovnickas and J. O'Farrill, Indirect, direct and collider detection of neutralino dark matter, JCAP 0408, 005 (2004) [arXiv:hep-ph/0405210].

[59] A. Arbey and F. Mahmoudi, SuperIso Relic: A program for calculating relic density and flavor physics observables in Supersymmetry, Comput. Phys. Commun. 181 (2010) 1277.

[60] G. Belanger, F. Boudjema, A. Pukhov and A. Semenov, micrOMEGAs2.0: A program to calculate the relic density of dark matter in a generic model, Comput. Phys. Commun. 176 (2007) 367.

[61] G. Belanger, F. Boudjema, A. Pukhov and A. Semenov, Dark matter direct detection rate in a generic model with micrOMEGAs2.1, Comput. Phys. Commun. 180, 747 (2009) [arXiv:0803.2360 [hep-ph]].

[62] G. Belanger, F. Boudjema, P. Brun, A. Pukhov, S. Rosier-Lees, P. Salati and A. Semenov, Indirect search for dark matter with micrOMEGAs2.4, [arXiv:1004.1092 [hep-ph]].

[63] E. Boos et al. [CompHEP Collaboration], CompHEP 4.4: Automatic computations from Lagrangians to events, Nucl. Instrum. Meth. A 534, 250 (2004) [arXiv:hep-ph/0403113].

[64] A. Pukhov, Calchep 2.3: MSSM, structure functions, event generation, and generation of matrix elements for other packages, [arXiv:hep-ph/0412191].

[65] T. Hahn, Generating Feynman diagrams and amplitudes with FeynArts 3, Comput. Phys. Commun. 140, 418 (2001) [arXiv:hep-ph/0012260].

[66] T. Hahn and M. Perez-Victoria, Automatized one-loop calculations in four and D dimensions, Comput. Phys. Commun. 118, 153 (1999) [arXiv:hep-ph/9807565].

[67] T. Hahn and J. I. Illana, Excursions into FeynArts and FormCalc, Nucl. Phys. Proc. Suppl. 160, 101 (2006) [arXiv:hep-ph/0607049].

[68] J. Alwall et al., MadGraph/MadEvent v4: The New Web Generation, JHEP 0709, 028 (2007).

[69] F. Maltoni and T. Stelzer, MadEvent: Automatic event generation with MadGraph, JHEP 0302, 027 (2003) [arXiv:hep-ph/0208156].

[70] T. Gleisberg et al., SHERPA 1.alpha, a proof-of-concept version, JHEP 0402, 056 (2004).

[71] M. Moretti, T. Ohl and J. Reuter, O'Mega: An optimizing matrix element generator, arXiv:hep-ph/0102195. 